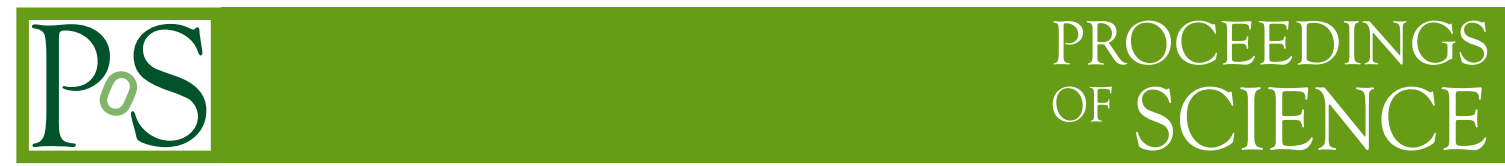

\title{
Higgs physics results by ATLAS and CMS
}

\author{
Nicolò Trevisani, for the ATLAS and CMS Collaboration ${ }^{a, *}$ \\ ${ }^{a}$ University of Oviedo - ICTEA, \\ Calle San Francisco, 3, 33003, Oviedo, Asturias (Spain) \\ E-mail: nicolo.trevisani@cern.ch
}

Since the discovery of the Higgs boson in 2012, significant steps forward in studying its properties have been made. Thanks to the huge amount of data, recent results measure not just the Higgs mass and total production cross-section in the so-called golden channels, but can also look at fiducial and differential measurements and search for rarer final states. The study of the Higgs pair production, fundamental to study the Higgs self-coupling, received a significant boost, too. This presentation focuses on the latest Higgs results published by the ATLAS and CMS collaborations using the entire dataset collected during the Run- 2 of the LHC.

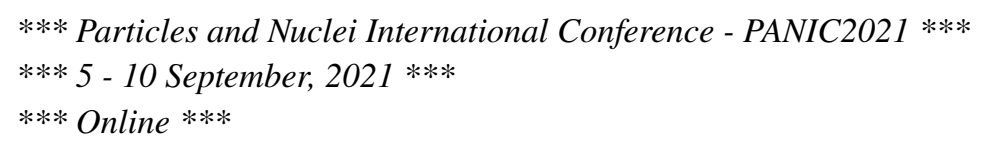

${ }^{*}$ Speaker 


\section{Introduction}

The Higgs boson is a fundamental piece of the Standard Model (SM) of particle physics, as it is responsible for the electroweak symmetry breaking and gives mass to vector bosons and fermions. At the Large Hadron Collider (LHC), the main Higgs production modes are gluon-gluon fusion $(\mathrm{ggF})$, vector-boson fusion $(\mathrm{VBF})$, and production in association with a vector boson $(\mathrm{VH})$ or with a top quark pair (ttH), shown in Figure 1 with the expected cross-sections.

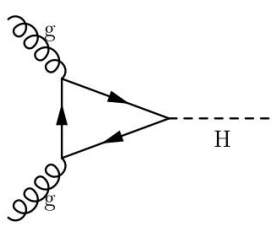

(a)

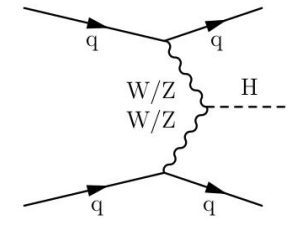

(b)

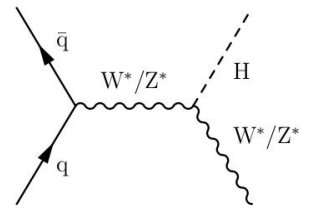

(c)

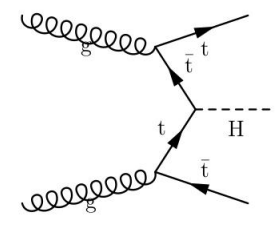

(d)

Figure 1: The four main Higgs production modes at the LHC, with their expected cross-sections at $13 \mathrm{TeV}$ : gluon-gluon fusion (48.6 pb), vector-boson fusion (3.8 pb), production in association with a vector boson $(2.3 \mathrm{pb})$, and production in association with a top-quark pair $(0.5 \mathrm{pb})$.

The first observation of the Higgs boson was announced by the ATLAS [2] and CMS [1] collaborations in 2012 [3-5]. Almost ten years later, the amount of data available has dramatically increased. On top of the Higgs boson mass [6,7] and total production cross-section, we can now measure many other properties and look for rare decays. The main production modes and final states, as decays to bosons and third-generation fermions, are studied in detail through fiducial and differential cross-section measurements. In addition, results are also presented in the simplified template cross-section framework (STXS) [8] to increase the re-interpretability of the precision measurements and minimize the theory dependence. Rarer final states are inspected, too, as the decays to pair of second-generation fermions, to a real and a virtual photon $\left(\mathrm{H} \rightarrow \gamma \gamma^{*}\right)$, to a $\mathrm{Z}$ boson and a photon $(\mathrm{H} \rightarrow \mathrm{Z} \gamma$ ), and the decay to invisible particles. Finally, we made a significant step forward in the study of the Higgs pair production, a fundamental process to study the Higgs self-coupling and the structure of the scalar Higgs field potential.

\section{The Golden Channels}

The $\mathrm{H} \rightarrow \mathrm{ZZ}$ and $\mathrm{H} \rightarrow \gamma \gamma$ final states are known as the golden channels, as they provide the best invariant mass resolution and played a fundamental role in the Higgs discovery.

The $\mathrm{H} \rightarrow \mathrm{ZZ}$ analyses select events with two pairs of opposite-sign, same-flavor leptons, resulting in a clean final state. Both ATLAS and CMS performed the cross-section measurement in a fiducial volume that closely matches the reconstruction-level selection to reduce the model dependence, and the results are provided in terms of integrated fiducial cross-section, differential cross-sections, and STXS [9-11].

The $\gamma \gamma$ final state, on the other hand, has to deal with an overwhelming background coming from the non-resonant production of photon pairs. Thus the results are obtained through a fit to data, where the good invariant mass resolution allows identifying the signal events, accumulated 
in a gaussian peak. Currently, both ATLAS and CMS have produced inclusive cross-section and STXS measurements using the full Run-2 dataset $[12,13]$, with ATLAS adding differential measurements [14].

Results are in agreement with the SM, as shown in Figure 2.

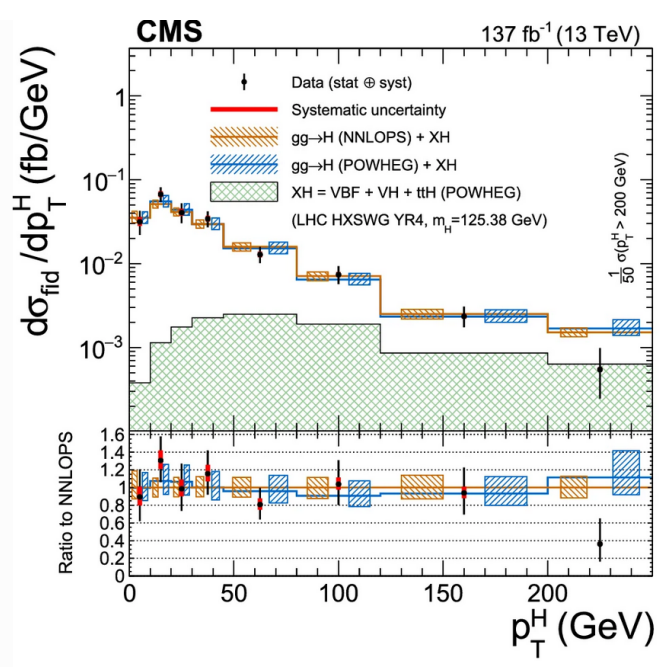

(a)

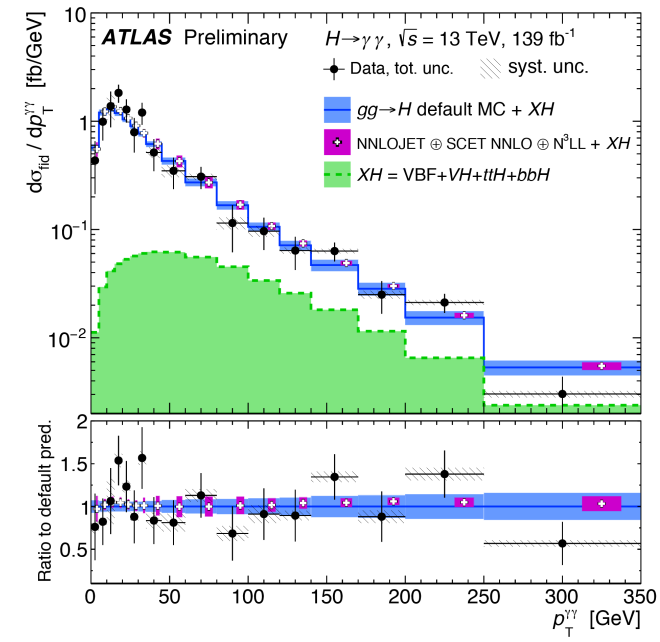

(b)

Figure 2: CMS differential cross-section measurement [11] as a function of the Higgs transverse momentum experiment in the $\mathrm{H} \rightarrow \mathrm{ZZ}$ final state (a) and ATLAS differential cross-section measurements [14] as a function of the Higgs boson transverse momentum in the $\mathrm{H} \rightarrow \gamma \gamma$ final state (b).

\section{The Other Main Channels}

The golden channels are not the only ones we can use to get precise measurements of the Higgs boson properties. The $\mathrm{H} \rightarrow \mathrm{WW}, \mathrm{H} \rightarrow \tau \tau$, and $\mathrm{H} \rightarrow$ bb decays do not provide an invariant mass resolution as good as those we have in the $\mathrm{ZZ}$ or $\gamma \gamma$ final states, but the high branching fractions allow us to get competitive results.

In the $\mathrm{H} \rightarrow \mathrm{WW}$ channel, final states with two opposite-sign leptons, coming from the decays of the $\mathrm{W}$ bosons, are selected. It allows to keep the background contamination, but the presence of neutrinos produced by the W bosons decay spoils the invariant mass resolution. While ATLAS published results in terms of total cross-section and STXS in 11 categories [15], CMS measured the integrated fiducial cross-section and the differential cross-section as a function of the Higgs transverse momentum and number of jets in the event [16].

To be orthogonal to the WW final state, in the $\mathrm{H} \rightarrow \tau \tau$ channel at least one of the two tau leptons is required to decay hadronically. As in the previous case, the invariant mass resolution is spoiled by the presence of neutrinos in the events. The results prepared by ATLAS include STXS measurement in 9 categories and inclusive cross-section [17], while CMS presented inclusive fiducial cross-section and differential cross-section measurements as a function of the number of jets in the event, Higgs transverse momentum, and transverse momentum of the leading jet in the event [18]. 
Finally, the $\mathrm{H} \rightarrow$ bb final state selects highly-boosted Higgs bosons, to reduce the contamination of non-resonant jets production. The two b jets thus merge into a single large-radius jet, representing the typical signature of this study. ATLAS [19] and CMS [20] produced results for the inclusive cross-section and differential cross-section as a function of the Higgs boson transverse momentum.

In all cases, the results are in good agreement with the SM expectations, as shown in Figure 3.

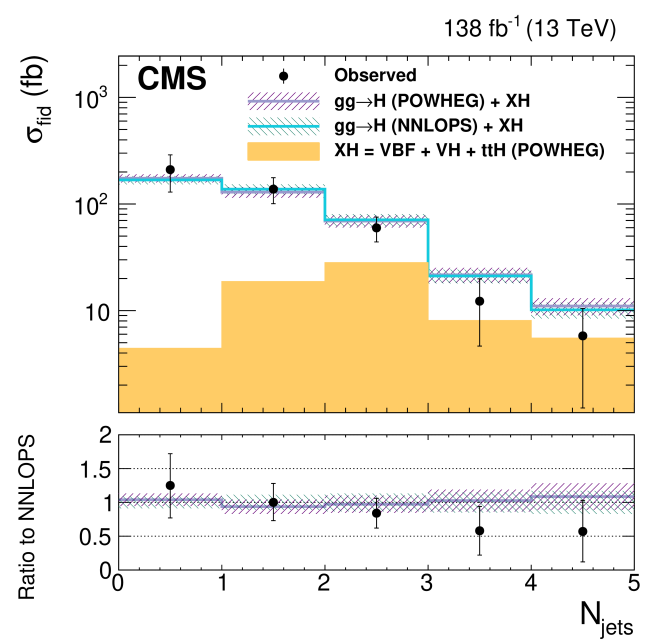

(a)

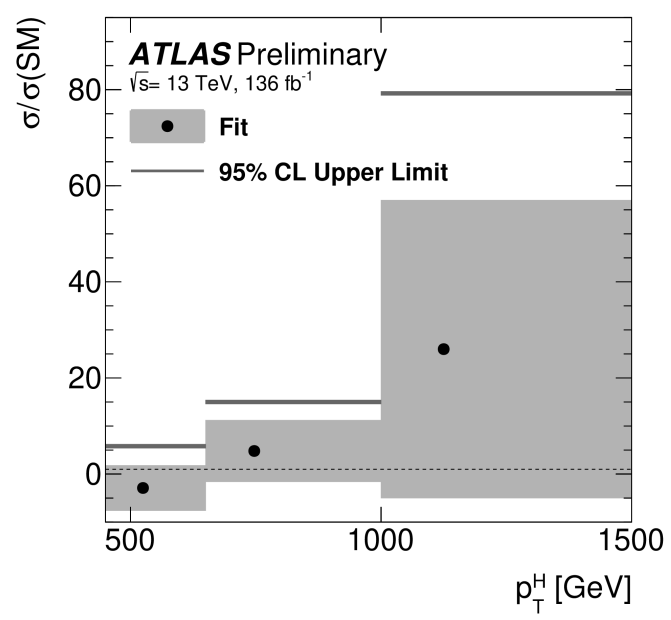

(b)

Figure 3: CMS differential cross-section measurements [18] as a function of the number of jets in the $\mathrm{H} \rightarrow \tau \tau$ channel (a) and ATLAS [19] differential cross-section results in the $\mathrm{H} \rightarrow$ bb final state (b).

\section{The Challenging Final States}

The decay channels that we describe in this section have small branching fractions so that it is still not possible to claim their observation. Nevertheless, using dedicated analysis techniques, we managed to show evidence of such processes or put competitive upper limits. They include Higgs decay to pair of second-generation fermions ( $\mathrm{H} \rightarrow \mu \mu$ and $\mathrm{H} \rightarrow \mathrm{cc}$ ), bosons $(\mathrm{H} \rightarrow \mathrm{Z} \gamma$ and $\mathrm{H} \rightarrow \gamma \gamma *)$, and invisible particles.

$\mathrm{The} \mathrm{H} \rightarrow \mu \mu$ final state provides the first evidence of the coupling of the Higgs boson to secondgeneration fermions. The signal is typically extracted using a fit to data to distinguish the signal peak above the dominant $\mathrm{Z} \rightarrow \mu \mu$ smoothly-falling invariant mass distribution. In addition, CMS uses a template-based approach for the vector boson fusion category to enhance sensitivity with a deep neural network. Both the experiments presented the results in terms of signal significance, with ATLAS observing a $2 \sigma$ excess [21] with respect to the background-only hypothesis and CMS a $3 \sigma$ excess [22].

The other final state involving second-generation fermions $(\mathrm{H} \rightarrow \mathrm{cc})$ has to deal with the large background due to multi-jet productions. The latest results, published only by the ATLAS collaboration using the full Run-2 dataset, use multivariate analysis techniques to identify jets produced by c quarks and target the $\mathrm{VH}$ associate production to trigger interesting events and suppress backgrounds $(\mathrm{ZH} \rightarrow v v \mathrm{cc}, \mathrm{WH} \rightarrow \ell v \mathrm{cc}$, and $\mathrm{ZH} \rightarrow \ell \ell \mathrm{cc})$. Due to the experimental challenges, the results 
are presented in terms of upper limits with respect to the SM expectations and are set to 26 times at a $95 \%$ confidence level [23].

$\mathrm{The} \mathrm{H} \rightarrow \mathrm{Z} \gamma$ and $\mathrm{H} \rightarrow \gamma \gamma^{*}$ channels target the same final state, with the $\mathrm{Z}$ boson or the virtual photon decaying to a pair of opposite-sign leptons. The signal is then extracted using a fit to the invariant mass of the $\ell \ell \gamma$ system in data. To target the $\mathrm{H} \rightarrow \gamma \gamma^{*}$ channel, events with low di-lepton invariant mass are selected, to veto the presence of a $\mathrm{Z}$ boson in the event. The selection is inverted in the analysis targeting the $\mathrm{H} \rightarrow \mathrm{Z} \gamma$ channel. Only the ATLAS collaboration produced results for these final states using the full Run- 2 datasets, managing to claim evidence for the $\mathrm{H} \rightarrow \gamma \gamma^{*}$ process, with an observed signal significance of $3.2 \sigma$, and obtaining a signal significance of $2.2 \sigma$ for the $\mathrm{H} \rightarrow \mathrm{Z} \gamma$ channel [24].

The interesting aspect of the Higgs boson decay to invisible particles is the possibility to reinterpret the results in terms of couplings with Dark Matter or other exotic particles. The latest results, provided by the ATLAS collaboration using the full Run-2 dataset, target the ZH (with $\mathrm{Z} \rightarrow \ell \ell$ ) associate production to trigger interesting events and rely on multivariate techniques to extract the signal. The results are presented in terms of upper limits of the branching fraction of the Higgs to invisible particles, corresponding to $18 \%$ at $95 \%$ confidence level. A combination with previous ATLAS results lowers the upper limit to $11 \%$ at $95 \%$ confidence level [25].

The main results of the challenging final states are summarized in Figure 4.

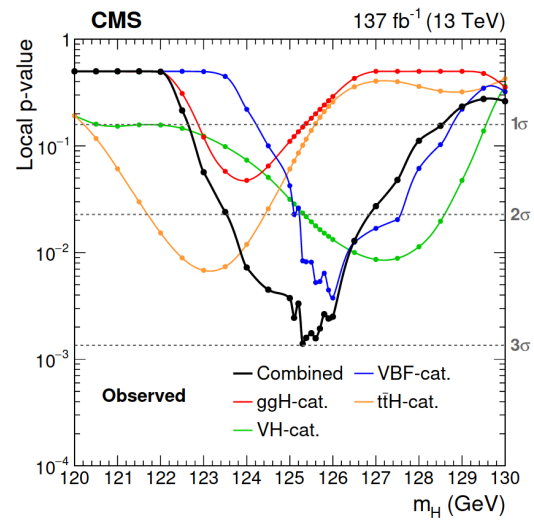

(a)

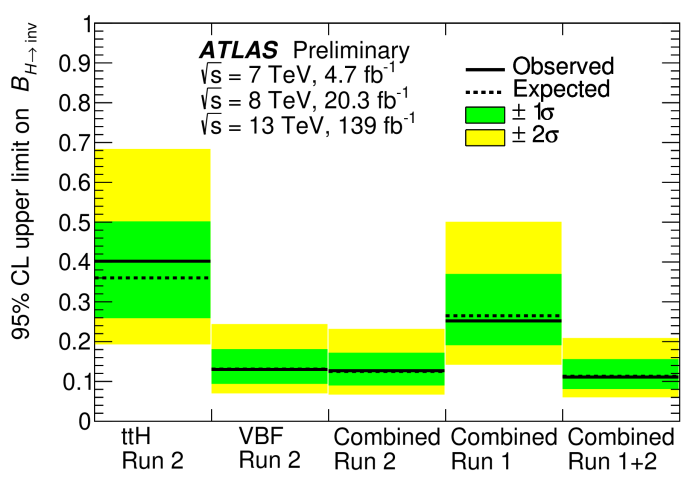

(b)

Figure 4: CMS [22] results observed p-values for the background-only hypotesis in the $\mathrm{H} \rightarrow \mu \mu$ analysis (a) and ATLAS [25] combination of upper limits on the Higgs branching fraction to invisible particles (b).

\section{The Double Higgs Production}

The double Higgs production opens the possibility of measuring the Higgs self-coupling $\left(\lambda_{\mathrm{HHH}}\right)$ and the couplings with vector bosons $\left(c_{V}\right.$ and $\left.c_{2 V}\right)$. The production modes at the LHC are shown in Figure 5, together with the expected production cross-section.

The main challenge in the study of this process is the low production cross-section, one order of magnitude with respect to those of single-Higgs production. The results are still limited by the small amount of available data, and the analyses focus on final states in which at least one of the Higgs bosons decays to a pair of b quarks, profiting for the large $\mathrm{H} \rightarrow \mathrm{bb}$ branching fraction (58\%). 


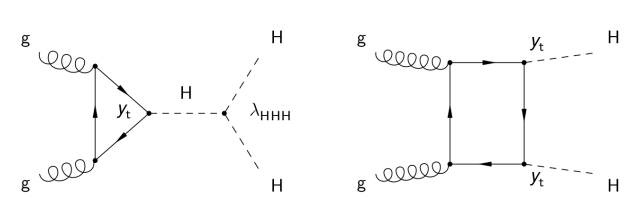

(a)
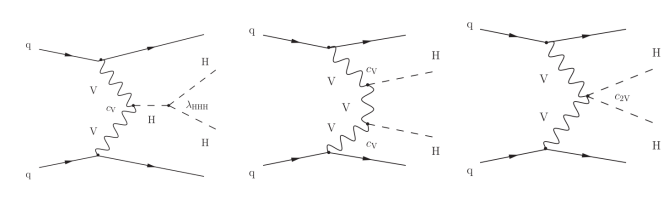

(b)

Figure 5: Left plots: feynman diagrams for the double Higgs production through gluon-gluon fusion $(31.1 \mathrm{fb})$. Right plots: feynman diagrams for the double Higgs production through vector-boson fusion $(1.726 \mathrm{fb})$. Note that the cross-sections are one order of magnitude lower with respect to those of singleHiggs production in Figure 1.

The $\mathrm{HH} \rightarrow$ bbbb channel presents the highest branching fraction (34\%) and targets both the ggF and VBF production mechanisms. To reject the dominant QCD and top backgrounds, a fit to a BDT discriminant or the invariant mass of the four b quarks is performed, depending on the targeted production mode. The most sensitive results, published by the CMS experiments [26], are interpreted as limits on the cross-section and couplings $\lambda_{\mathrm{HHH}}$ and $c_{2 V}$, expressed in terms of the ratio with the SM expectation ( $\kappa_{\lambda}$ and $\kappa_{2 V}$, respectively):

- $\sigma(\mathrm{pp} \rightarrow \mathrm{HH} \rightarrow 4 \mathrm{~b})<3.6(7.3) \times \mathrm{SM}$ obs. (exp.) at $95 \%$ confidence level

$-2.3<\kappa_{\lambda}<9.4$ at $95 \%$ confidence level

$-0.1<\kappa_{2 V}<2.2$ at $95 \%$ confidence level

A separate result, produced by the CMS experiment [28], targets the non-resonant VBF doubleHiggs production to measure $\kappa_{2 V}$. Events with two large-radius jets, each of them produced by the decay of a Higgs to pair of b quarks, are selected. A multivariate classifier based on graph convolutional networks and mass regression is used to reject the leading top and QCD backgrounds, managing the most sensitive results on $\kappa_{2 V}: 0.6<\kappa_{2 V}<1.4$ at $95 \% \mathrm{CL}$.

The final state in which one Higgs decays to a pair of $b$ quarks and the other to a pair of photons has a small branching fraction but provides a clean final state and good resolution. The latest results, published by the ATLAS collaboration, use a BDT discriminant and invariant mass categorization to separate the signal from main backgrounds [30]. They represent the most stringent limits on the $\kappa_{\lambda}$ to date: $-1.5<\kappa_{\lambda}<6.7$ at $95 \%$ confidence level.

Finally, the $\mathrm{HH} \rightarrow \mathrm{bb} \tau \tau$ final state is a good compromise between branching fraction and background contamination. The ATLAS collaboration [31] published a search optimized to maximize the sensitivity to the measurement of the cross-section, obtaining a competitive upper limit of 4.7 times with respect to the SM expectation, at a 95\% confidence level.

The most stringent limits on $\kappa_{\lambda}$ and $\kappa_{2 V}$, provided by the boosted $\mathrm{HH} \rightarrow \mathrm{bbbb}$ and $\mathrm{HH} \rightarrow \mathrm{bb} \gamma \gamma$ searches, are shown in Figure 6.

\section{Summary}

We have presented the recent Higgs results published by the ATLAS and CMS collaboration using the full Run-2 dataset. The golden channels, decays to vector bosons, and third-generation 


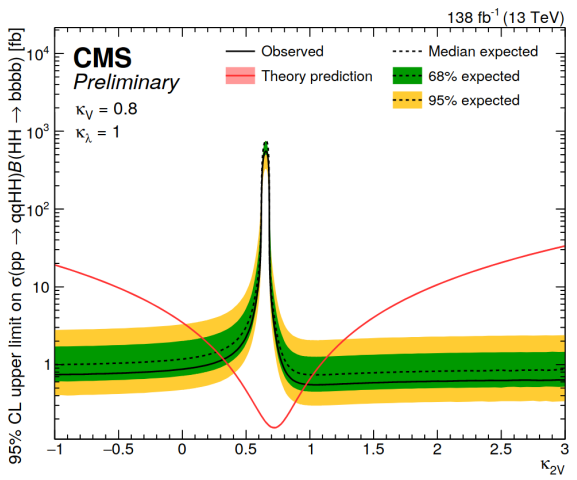

(a)

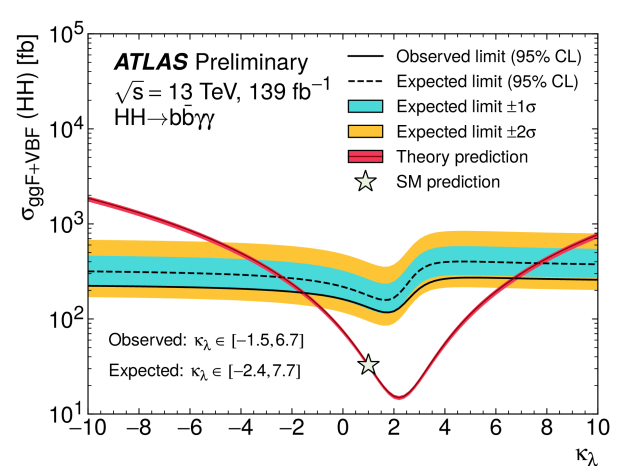

(b)

Figure 6: The most stringent limits on the $\kappa_{2 V}$ [28] coupling (a) and on the $\kappa_{\lambda}$ coupling [30].

fermions are well studied, through inclusive, fiducial, and STXS measurements. A great effort has been made to explore decays to second-generation fermions and other rare final states $\left(\gamma \gamma^{*}\right.$ and $\mathrm{Z} \gamma$ ). Finally, limits on the double Higgs production cross-section and the Higgs self-coupling are more stringent and already close to SM expectations. The forthcoming Run 3 will help improve the current results and prepare the high-luminosity phase.

\section{References}

[1] CMS Collaboration, JINST 3 (2008) S08004, 961, doi:10.1088/1748-0221/3/08/S08004

[2] ATLAS Collaboration, JINST 3 (2008) S08003, doi: 10.1088/1748-0221/3/08/S08003

[3] CMS Collaboration, Phys. Lett., vol. B716, pp. 30-61, 2012

[4] CMS Collaboration, J. High Energ. Phys., vol. 2013, art. 81, 2013. https://doi.org/10.1007/JHEP06(2013)081

[5] ATLAS Collaboration, Phys. Lett., vol. B716, pp. 1-29, 2012

[6] CMS Collaboration, Phys. Lett., vol. B805, 135425, 2020

[7] ATLAS Collaboration, ATLAS-CONF-2020-005, http://cds.cern.ch/record/2714883

[8] Nicolas Berger et al. arXiv:1906.02754

[9] ATLAS Collaboration, Eur. Phys. J. C, vol. 80, 942, 2020

[10] ATLAS Collaboration, Eur. Phys. J. C, vol. 80, 957, 2020

[11] CMS Collaboration, Eur. Phys. J. C, vol. 81, 488, 2021

[12] CMS Collaboration, J. High Energ. Phys., vol. 2021, art. 27, 2021. https://doi.org/10.1007/JHEP07(2021)027

[13] ATLAS Collaboration, ATLAS-CONF-2020-026, https://cds.cern.ch/record/2725727 
[14] ATLAS Collaboration, ATLAS-CONF-2019-029, http://cdsweb.cern.ch/record/2682800

[15] ATLAS Collaboration, ATLAS-CONF-2021-014, http://cdsweb.cern.ch/record/2759651/

[16] CMS Collaboration, J. High Energ. Phys., vol. 2021, art. 3, 2021, https://doi.org/10.1007/JHEP03(2021)003

[17] ATLAS Collaboration, ATLAS-CONF-2021-044, https://atlas.web.cern.ch/Atlas/GROUPS/PHYSICS/CONFNOTES/ATLAS-CONF-2021044/

[18] CMS Collaboration, CMS-HIG-20-015, arXiv:2107.11486

[19] ATLAS Collaboration, ATLAS-CONF-2021-010, https://cds.cern.ch/record/2759284

[20] CMS Collaboration, J. High Energ. Phys., vol. 2020, art. 85, 2021, https://doi.org/10.1007/JHEP12(2020)085

[21] ATLAS Collaboration, Phys. Lett., vol. B812, 135980, 2021

[22] CMS Collaboration, J. High Energ. Phys., vol. 2021, art. 148, 2021, https://doi.org/10.1007/JHEP01(2021)148

[23] ATLAS Collaboration, ATLAS-CONF-2021-021, https://atlas.web.cern.ch/Atlas/GROUPS/PHYSICS/CONFNOTES/ATLAS-CONF-2021021/

[24] ATLAS Collaboration, Phys. Lett., vol. B819, 136412, 2021

[25] ATLAS Collaboration, ATLAS-CONF-2020-052, http://cdsweb.cern.ch/record/2743055/

[26] CMS Collaboration, CMS-PAS-HIG-20-005, https://cds.cern.ch/record/2771912

[27] ATLAS Collaboration, J. High Energ. Phys., vol. 2020, art. 108, 2020, https://doi.org/10.1007/JHEP07(2020)108

[28] CMS Collaboration, CMS-PAS-B2G-21-001, https://cds.cern.ch/record/2776802

[29] CMS Collaboration, J. High Energ. Phys., vol. 2021, art. 257, 2021, https://doi.org/10.1007/JHEP03(2021)257

[30] ATLAS Collaboration, ATLAS-CONF-2021-016, https://atlas.web.cern.ch/Atlas/GROUPS/PHYSICS/CONFNOTES/ATLAS-CONF-2021$016 /$

[31] ATLAS Collaboration, ATLAS-CONF-2021-030, https://cds.cern.ch/record/2777236/ 